\title{
REGRESIÓN LOGISTICA EN LA EVALUACIÓN DE LA ESPORULACIÓN DE MICORRIZAS EN PASTO Bothriochloa pertusa (L) A. Camus.
}

\section{LOGISTIC REGRESSION IN ASSESSING THE SPORULATION OF MYCORRHIZAE IN Bothriochloa pertusa (L) A. Camus PASTURE.}

\author{
PEREZ C., ALEXANDER. Dr, ${ }^{1 *}$ FUENTES C., JUSTO. Esp. ${ }^{2}$
}

${ }^{1}$ Universidad de Sucre, Facultad de Ciencias Agropecuarias, Colombia. Grupo Bioprospección Agropecuaria. ${ }^{2}$ Universidad de Sucre, Colombia, Facultad de Ingeniería, *Correspondencia:* alexander.perez@unisucre.edu.co

\section{Resumen}

El objetivo del presente trabajo fue obtener una función logística o función de probabilidad que permitiera medir las posibilidades de ocurrencia del evento esporulación de hongos formadores de micorrizas arbusculares nativos, asociados al pasto Bothriochloa pertusa (L) A. Camus, localizas en fincas ganaderas de cuatro zonas agroecológicas, municipio de Corozal, Colombia, como respuesta a la actividad de ciertos factores químicos del suelo tales como: $\mathrm{pH}$, Materia Orgánica, Fósforo, Potasio. Las muestras de suelo fueron colectadas en fincas ganaderas sembradas con pasto colosuana. El aislamiento, el conteo de esporas/ $100 \mathrm{~g}$ de suelo y la identificación de los hongos fue realizadas en el laboratorio de microbiología de la Universidad de Sucre. El modelo o función de probabilidad creada fue utilizado para medir las posibilidades de ocurrencia de la esporulación como respuesta a la presencia de ciertos factores químicos del suelo. Para obtener esta función logística se determinó la importancia de cada uno de estos factores de riesgo y sus posibles interacciones. Fueron muestreadas 48 fincas ganaderas distribuidas en cuatro zonas agroecológicas diferentes, la cantidad esporas/ 100 de suelo, oscilo entre (900) 2600 - 4000 (7300); se aislaron 31 morfotipos nativos de hongos formadores de micorrizas arbusculares distribuidos en dos géneros Glomus y Gigospora, predominando en su mayoría especies del género Glomus. El proceso de modelación logística encontró que los factores que mejor explican el fenómeno de la esporulación de hongos formadores de micorrizas asociados a rizosfera de pasto colosuana fueron el $\mathrm{pH}$ y el Potasio.

Palabras claves: Micorrizas, esporas, suelo, modelación logística 


\section{Abstract}

The purpose of this study was to obtain a logistic function or probability function to measure the chances of occurrence of the events sporulation of native arbuscular mycorrhizal-creater fungi associated with the grass Bothriochloa pertusa (L) A. Camus, located in four agro-ecological zones cattle farms, in the municipality of Corozal, Colombia, in response to the activity of certain soil chemical factors such as: $\mathrm{pH}$, Organic Matter, Phosphorus, Potassium. Soil samples were collected in cattle farms planted with Colosoana grass. The isolation, the counting of soil spores / $100 \mathrm{~g}$ and the identification of fungi were carried out in the microbiology laboratory at the University of Sucre. The model created or probability function was used to measure the chances of occurrence of sporulation in response to the presence of certain chemical factors of soil. To obtain this logistic function, the importance of each of these risk factors was determined and their possible interactions. 48 cattle farms were sampled on four different agro-ecological zones, the amount of soil spores / 100, ranged from (900) from 2600 to 4000 (7300). 31 native morphotypes of arbuscular mycorrhizal-creating fungi were isolated, by distributing them in two types Glomus and Gigospora, being predominant the Glomus species. The logistic modeling process found that the factors that best explain the phenomenon of sporulation of mycorrhizal-creating fungi associated with Colosoana grass rhizospheres were $\mathrm{pH}$ and potassium.

Key words: Mycorrhizal, spores, soil, logistic modelling

\section{Introducción}

En la región Caribe Colombiano se encuentra el mayor hato ganadero del país, aproximadamente $35 \%$ del total nacional, siendo su casi exclusiva fuente de alimentación el pastoreo de gramíneas y leguminosas nativas o introducidas, lo cual indica que la productividad animal por unidad de superficie está, en gran medida, determinada por el valor nutritivo y la producción de materia seca del forraje en oferta (AGUILERA, 2005).

Se estima que el pasto colosuana o kikuyina (Bothriochloa pertusa) (L). A. Camus, alcanza un total de 274.005 Hectáreas $(\mathrm{Ha})$, distribuidas en 19 municipios del departamento de Sucre (UNIVERSIDAD DE SUCRE, 1996). El municipio de Corozal cuenta con la mayor área sembrada con esta especie de pasto en la 
región $(32.223 \mathrm{Ha})$.La ganadería de doble propósito, en el departamento de Sucre, representa un $84.9 \%$ de su territorio (DANE, 2000). La importancia de la alimentación animal en el departamento radica en el valor nutritivo del pasto que es determinante en la productividad animal. Una de las causas de escasez o falta total de forraje durante la época seca en el departamento de Sucre, se debe a la estacionalidad de las lluvias. Esta escasez es afectada cuando los suelos presentan diferentes grados de compactación, problemas erosivos y niveles bajos de fertilidad, y cuando no se realizan abonamientos. Además de esto, cuando se practica el pastoreo extensivo, lo que genera la degradación de las praderas (AGUILERA, 2005).

Los hongos micorrízicos arbusculares son simbiontes fisiológicamente obligados, debido a que su ciclo biológico se da únicamente cuando están asociados a una especie vegetal. Tanto los hongos como las plantas tienen distribución universal, presentándose de esta manera ecotipos adaptados a condiciones diversas y extremas. Es de señalar que las plantas y las micorrizas tienen un origen común, además existen evidencias en registros fósiles que las plantas estaban micorrizadas, se acepta que la simbiosis ha coevolucionado y se ha perpetuado con la propia planta (SYLVIA, 1999). Las micorrizas arbusculares están ampliamente distribuidas en condiciones naturales, se encuentran en todos los continentes, excepto en la Antártida; se dan en todos los suelos, incluyendo los de minas abandonadas, suelos agrícolas, suelos de pantanos y en hábitat acuáticos (AZCON y BAREA, 1980; BOLAN, 1991; CORWELL, 2001; TANG et al., 2001)

Las micorrizas arbusculares son un importante factor biológico dentro de la estructura y funcionamiento de los suelos, e inciden sobre el comportamiento ecológico, productividad y composición de comunidades vegetales naturales, así como de cultivos agrícolas y plantaciones forestales. Los hongos formadores de micorrizas arbusculares son considerados, como parte de la diversidad biológica de los suelos y deben ser incluidos tanto en los inventarios como en los análisis de 
la biodiversidad a nivel de ecosistemas y agroecosistemas (GUERRERO, 1996; VAN DER HEISDEN, 1998; BAREA, 1998; JEFFRIES y BAREA, 1999).

El establecimiento de la simbiosis va a depender de las interacciones entre los tres componente del sistema: el hongo, la planta y las condiciones ambientales. Su presencia implica que ocurran procesos de reconocimiento entre los simbiontes, compatibilidad y especificidad, los cuales condicionan su expresión y conducen a la integración morfológica y funcional de las asociaciones (SÁNCHEZ, 1999). La esporulación de los hongos formadores de micorrizas arbusculares bajo condiciones de campo están determinado por: las condiciones físico-químicas del suelo $(\mathrm{pH}$, contenido de fósforo, temperatura, aireación, textura y contenido de materia orgánica), condiciones climáticas (intensidad y duración de la luz, temperaturas, humedad, épocas de lluvias y épocas secas) y por las prácticas agronómicas (preparación del terreno, aplicación de pesticidas y prácticas culturales) (GONZALEZ, 1996).

Existen escasas investigaciones que permitan evaluar en forma integral, parámetros físico, químicos y biológico, buscando determinar sus efectos sobre la calidad de un ecosistema y los que existen son pocos conclusivos y de difícil interpretación, dada la gran complejidad del sistema en estudio en donde se evalúan varias características, generando un número mayor de datos, lo que proporcionalmente dificulta su interpretación. Una estrategia para evaluar el conjunto de indicadores biológicos en la calidad de los ecosistemas comúnmente sugeridos es el análisis a través de herramientas de estadística robusta que permita la conversión e interpretación de un conjunto de variables con una alta correspondencia entre ciertos indicadores y un componente, resultando en un alto peso absoluto del indicador en un componente determinado (TOTOLA y CHAER, 2002) 
Las evidencias que existen acerca de la importancia de la simbiosis micorrizas arbusculares y plantas, relacionadas con el mejoramiento de las condiciones físico-químicas del suelo; la estimulación del crecimiento y el mejoramiento de la calidad nutricional de las especies vegetales, convirtiéndolas en más tolerantes a condiciones adversas tanto abióticas como bióticas (AZCON y BAREA, 1980; SIEVERDING, 1984; BOLAN, 1991; BAGO y AZCON, 1998; SALAMANCA, 1999; BAREA, 2002), y la necesidad de elucidar un modelo que permitiera conceptualizar probabilísticamente la relación existente entre la densidad de esporas de estos hongos con las propiedades físico-químicas del suelo, son hechos que justifican la profundización de estudio de esos componentes biológicos con relación al ecosistema.

El presente trabajo plantea un modelo de predicción del evento de esporulación de de hongos formadores de micorrizas arbusculares con relación a parámetros físico químicos del suelo a nivel de fincas ganaderas, bajo las condiciones del municipio de Corozal.

\section{Materiales y métodos}

\section{Área de estudio}

El área de estudio fue realizado en el Municipio de Corozal, localizado al Noreste del departamento de Sucre, Colombia, a los $8^{\circ} 55^{\prime}$ y $9^{\circ} 19^{\prime \prime}$ de latitud Norte, y entre $75^{\circ} 25^{\prime \prime}$ y $74^{\circ} 42^{\prime \prime}$ al Este del meridiano de Greenwich. Tiene una extensión de 20.333 hectáreas aproximadamente, cuenta con 5100 Has con pendientes entre 0 y $3 \%, 5113.94$ Has entre 3 y $7 \%, 2528$ Has entre 12 y $25 \%$ y 7586.24 Has con más de $25 \%$. Una altura que oscila entre 174 a 200 metros sobre el nivel del mar, temperatura promedio de $28^{\circ} \mathrm{C}$, precipitación media anual de $1105 \mathrm{~mm}$ y una humedad relativa promedio anual del $80 \%$. El municipio de Corozal pertenece a la caracterización fisiográfica de Sabanas que constituye el declive general de los montes de María hacia la depresión del bajo Cauca y San Jorge. 


\section{Muestreo.}

Las muestras fueron colectadas en el primer semestre de 2007, en fincas ganaderas establecidas únicamente con el pasto colosuana o kikuyina (Bothriochloa pertusa (L) A. Camus) en el municipio de Corozal, ubicadas según el PLAN DE ORDENAMIENTO TERRITORIAL (2002) y el INSTITUTO GEOGRÁFICO AGUSTÍN CODAZZI (1998), en cuatro zonas agrologicas según la clasificación de tierras por su capacidad de uso y manejo, en las subclases: III sc - Iv hs; VIIsc - Iv esc; VI sc - VI esc y VII sc - VII esc. En las fincas ganaderas se realizó un muestreo, representativo, tomando entre 15 a 20 submuestras al azar a una profundidad de $0-20 \mathrm{~cm}$, recolectando al tiempo suelo y raíces. Las submuestras se homogenizaron para conformar una muestra por finca con un peso aproximado de 2000 gramos, las cuales se depositaron en bolsas plásticas rotuladas con el número de la finca, corregimiento, área sembrada con el pasto y fecha de recolección. La muestra tomada de cada finca ganadera se dividió en dos porciones iguales de 1 kilogramo para realización de análisis físico-químico y microbiológico.

\section{Procesamiento de muestras y aislamiento de esporas.}

Las muestras de suelos fueron tamizadas para separar las partes gruesas de suelos (piedras, cascajos) y raíces. Una vez tamizadas se procedió al análisis físico-químico y al aislamiento de esporas de hongos formadores de micorrizas arbusculares, mediante técnica propuesta por SIEVERDING (1983), DANIELS y SKIPPER (1991), BOTERO (1998).

El conteo de esporas se realizo tomando dos mililitros de cada muestra, fue depositadas en cámara cuenta nematodos (advance equine products 5004-228th Avenue SI Issaquah, Washington 98029 USA) (MENGE y TIMMER, 1991) y se realizó tres conteos por muestra para obtener un estimativo del número total de esporas en 100 gramos de suelos por finca. De cada conteo respectivo, el contenido de la cámara se recolectó en cajas de petri, se observó al 
estereoscopio y con la ayuda de una aguja de disección se reunieron morfotipos, teniendo en cuenta similitud de forma, color y tamaño de las esporas. Con la ayuda de una micropipeta se extrajeron las esporas de los diferente morfotipos encontrados, se colocaron en tubos de ensayos con agua estéril, se rotularon con el número de la finca donde fueron aislados y se guardaron en nevera a $4^{\circ} \mathrm{C}$ durante 3 días, para su posterior identificación. Los morfotipos aislados se depositaron en cajas de petri, se observaron al estereoscopio para detallar sus características en agua, verificar y eliminar esporas de otros morfotipos y partículas contaminantes. Una vez limpias las esporas y verificado el morfotipo, se realizó la identificaron a nivel de géneros, mediante técnicas propuestas por SCHENCK y PÉREZ (1990), MORTON (1996) e INVAM (2002).

\section{Regresión logística.}

Se realizó un análisis de regresión logística múltiple con la variable dicotómica ESPORULACION (SI/NO) como variable explicada de las siguientes variables regresoras candidatas: ZONAS $\left(\mathbf{X}_{\mathbf{1}}\right)$, Categórica $\mathbf{P H}$ Acidez, $\left(\mathbf{X}_{2}\right)$, Métrica MO (Materia Orgánica), $\left(\mathbf{X}_{3}\right)$, Métrica $\mathbf{P}$ (Fósforo), $\left(\mathbf{X}_{4}\right)$, Métrica $\mathbf{K}$ (Potasio), $\left(\mathbf{X}_{5}\right)$, Métrica. De esta manera se estimó, ¿qué probabilidad tiene un suelo de realizar ESPORULACION o no? Se observaron, desde un punto de vista microbiológico, las variables regresoras que el proceso de modelación (mediante Regresión Logística Múltiple) seleccionó como las que mejor explican la variabilidad de la esporulación (HORMER y LEMESHOW 1989; HAIR et al., 1998).

\section{Resultados}

\section{Zona de estudio.}

Se muestrearon 43 fincas ganaderas distribuidas en cuatro zonas agrologicas diferentes pertenecientes al municipio de Corozal, las cuales de acuerdo a los resultados de los análisis físico-químicos de suelos de cada zona, reportó valores de $\mathrm{pH}$ que oscilaron de fuertemente ácidos a moderadamente alcalinos, los contenidos de fósforo, materia orgánica y nitrógeno se encuentran de bajos a 
medios y los valores de calcio, magnesio y potasio, presentan rangos de medios a altos.

\section{Densidad de esporas y población de micorrizas.}

La densidad de esporas/100 $\mathrm{g}$ de suelos de hongos formadores de micorrizas arbusculares, oscilaron en un rango de (1300-) 1900 (-2500) para la zona 1; (2600-) 3500 (-4400), Zona 2; (900-) 3550 (-6200), zona 3 y (4000-) 5650 (-7300), zona 4 . Se observa que los mayores valores encontrados de esporas se encuentran la zona agroecológica cuatro, mientras que los valores mínimos se reportan para la Zona 1 (Tabla 1 ).

Tabla 1. Densidades de esporas/ $100 \mathrm{~g}$ de suelos de hongos formadores de micorrizas arbusculares en fincas ganaderas del municipio de corozal, Colombia.

\begin{tabular}{|c|c|}
\hline Zona Agrológica & $\begin{array}{c}\text { Valor mínimo, promedio y } \\
\text { máximo de esporas/100 g de } \\
\text { suelos }\end{array}$ \\
\hline 1 & $(1300-) 1900(-2500)$ \\
\hline 2 & $(2600-) 3500(-4400)$ \\
\hline 3 & $(900-) 3550(-6200)$ \\
\hline 4 & $(4000-) 5650(-7300)$ \\
\hline
\end{tabular}

En los suelos muestreados, fueron aislados 31 morfotipo, con características microscópica, similares a especies del géneros Glomus y Gigaspora, descritas por (SCHENCK y PEREZ, 1990). El mayor número de morfotipos encontrados fueron reportados en la zona agrologica 1 y los menores en valores en la zona 2 y 3 (figura 1). 


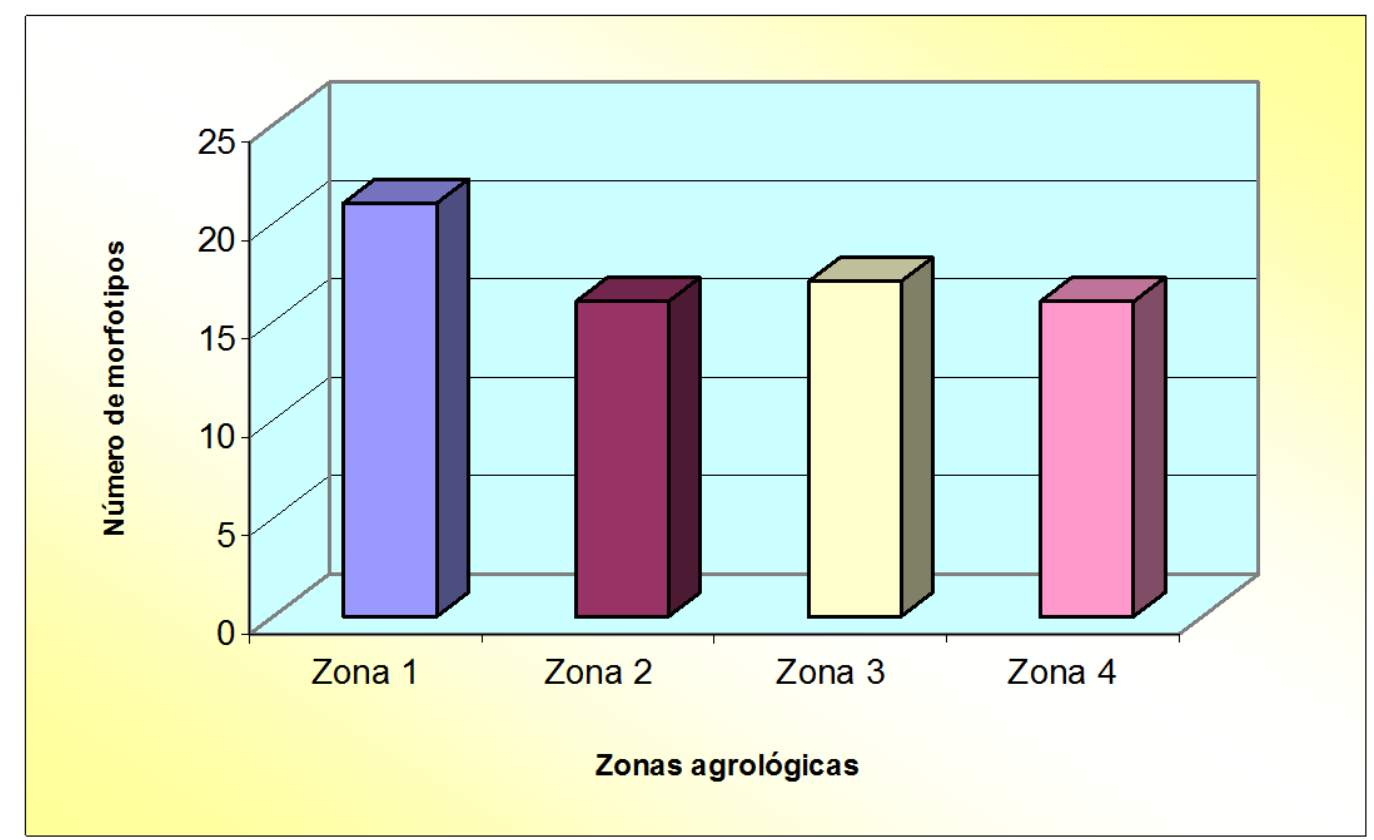

Figura 1. Relación de morfotipos aislados de muestras de suelos de fincas ganaderas por zonas agrológicas en el municipio de Corozal.

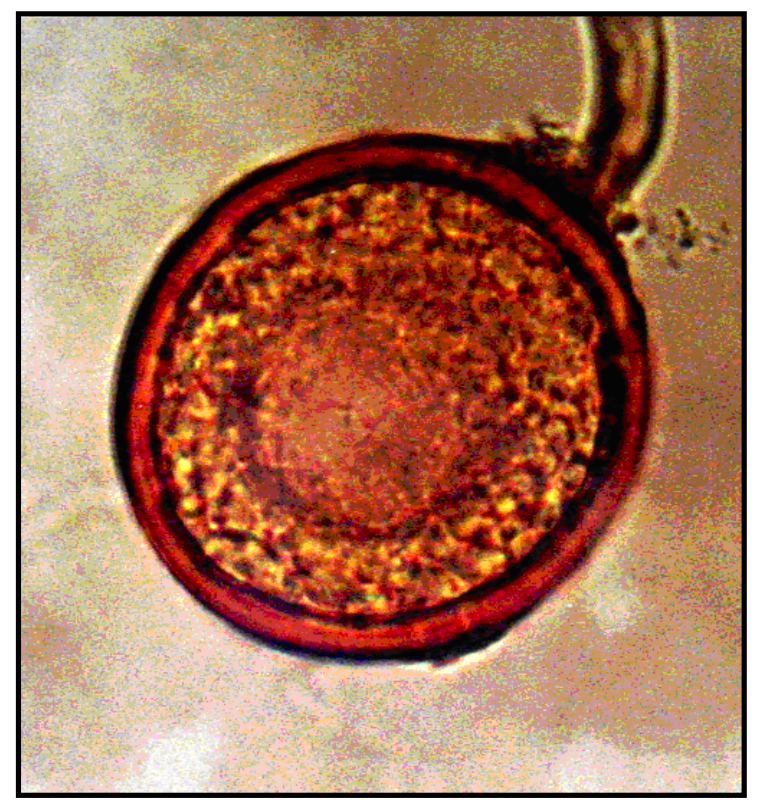

Figura 2. Morfotipo del genero Glomus, cf Glomus invermayanum Hall, 1977(40 $X$ ), encontrado en suelos de fincas ganaderas del municipio de Corozal, Colombia. 


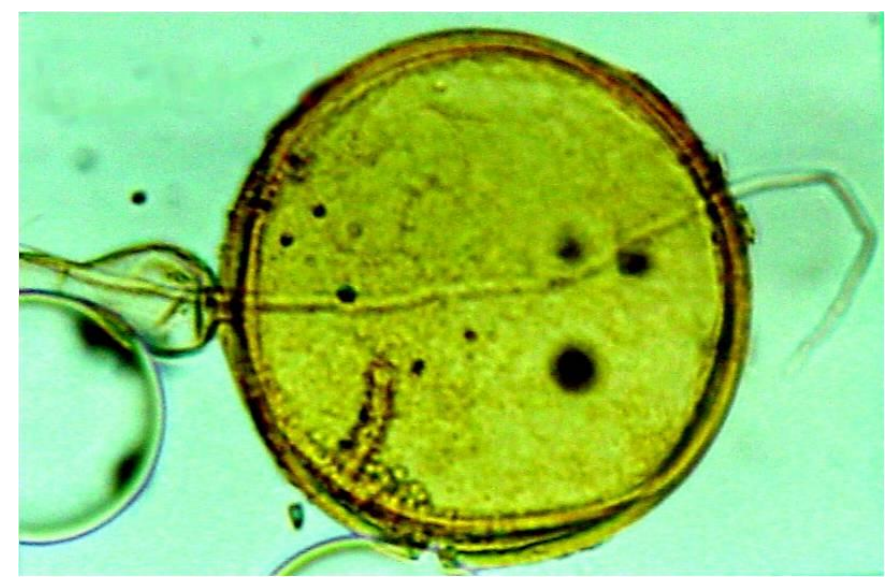

Figura 3. Morfotipo del género Gigaspora, cf Gigaspora rosea Nicolson \& Schenck, 1979 ( 40x), encontrado en suelos de fincas ganaderas del municipio de Corozal, Colombia.

Modelo definitivo obtenido en el proceso de modelación por pasos hacia atrás

En la tabla 2 se muestra el resumen de los resultados obtenidos en el modelo logístico, el cual reporta a las variables $\mathrm{pH}$ y $\mathrm{K}$ como las variables que mejor explican la variación del evento esporulación

Tabla 2. Modelación final de variables químicas que explican el evento de esporulación de hongos formadores de micorrizas arbusculares.

\begin{tabular}{|c|c|c|c|c|c|r|}
\hline Modelo & B & E.T. & Wald & gl & Significancia & \multicolumn{1}{|c|}{ Exp(B) } \\
\hline PH & -2.879 & 1.008 & 8.148 & 1 & 0.004 & 0.056 \\
\hline K & 5.077 & 2.427 & 4.378 & 1 & 0.036 & 160.363 \\
\hline Constante & 15.048 & 5.795 & 6.744 & 1 & 0.009 & 3429749.085 \\
\hline
\end{tabular}

En la Tabla 2, se aprecia que todos los valores del estadístico de Wald son superiores a 2 indicando que los coeficientes obtenidos por el modelo son significativamente diferentes de cero. La siguiente es la ecuación ajustada a partir de los coeficientes del modelo definitivo: 


$$
\ln \left[\frac{\hat{p}}{1-\hat{p}}\right]=15.048-2.879 * P H+5.077 * K
$$

Figura 4. Ecuación para la modelación de la función logísticas de las variables $\mathrm{pH}$ y $\mathrm{K}$ y el evento de esporulación de micorrizas arbusculares en suelo ganaderos del municipio de Corozal.

\section{Discusión}

Durante los últimos 20 años, en los suelos de las fincas ganaderas, pertenecientes al municipio de Corozal, no se ha realizado ninguna práctica agrícola para mejorar las propiedades físico-químicas del suelo, principalmente su aireación, ni se realiza abonamiento para suplir la extracción de nutrientes que la especie de pasto ha venido haciendo a través del tiempo. A pesar de esta serie de limitaciones edafológicas y de manejo, la especie de pasto Bothriochloa pertusa (L) A. Camus, presenta algunas características deseables para el empresario ganadero como son: rápida recuperación después del pastoreo, especie altamente estolonifera, alta producción de semillas, resistencia al pisoteo y buena gustosidad para el ganado.

Los rangos de densidad de esporas $/ 100 \mathrm{~g}$ de suelos hallados en estas fincas ganaderas, son mayores a los valores registrados por PICONE (2000), quien reporta un rango de 830 - 2600 esporas $/ 100 \mathrm{~g}$ de suelos en estudio realizados con pasturas en Nicaragua y Costa Rica. La abundancia de esporas/100 g de suelos encontrados en el presente estudio, probablemente fue debido a las condiciones de la época de muestreo, ya que en el momento de la recolección la zona se encontraba en época seca prolongada. Esto corrobora lo planteado por BONILLA(2000), en su investigación sobre utilización de hongos micorrízicos arbusculares en la producción agrícola realizada en el departamento del Cesar con varias especies de cultivos comerciales, quien afirma que en verano las plantas están bajo condición de estrés, el déficit de agua estimula la producción de esporas. Similarmente, esta hipótesis es concordante por lo expuesto por 
GUERRERO(1996), que señala que un déficit en el suministro de agua crea una situación adversa que estimula la producción de esporas durante la época seca y conduce a la adaptación de las micorrizas arbusculares a esa condición de estrés hídrico en el suelo.

De los 31 morfotipos aislados, el $96.9 \%$ corresponden a especies del género Glomus y $3.1 \%$ al género Gigaspora (Véase la Figura 1). Lo que indica, que existe menor diversidad de géneros de hongos formadores de micorrizas arbusculares asociados a la especie Bothriochloa pertusa (L) A. Camus; bajo las condiciones edafoclimáticas de las fincas ganaderas en el municipio de Corozal. Por las características de los morfotipos encontrados, se observa que existe una gran diversidad de especies del género Glomus y de acuerdo a los resultados hallados por AHN-HEUM (2001), LUGO y CABELLO (2002), quienes confirman que el pastoreo continuo produce disminución de la diversidad de especies de micorrizas arbusculares e incrementa la tolerancia y adaptación de ciertas especies a estas condiciones.

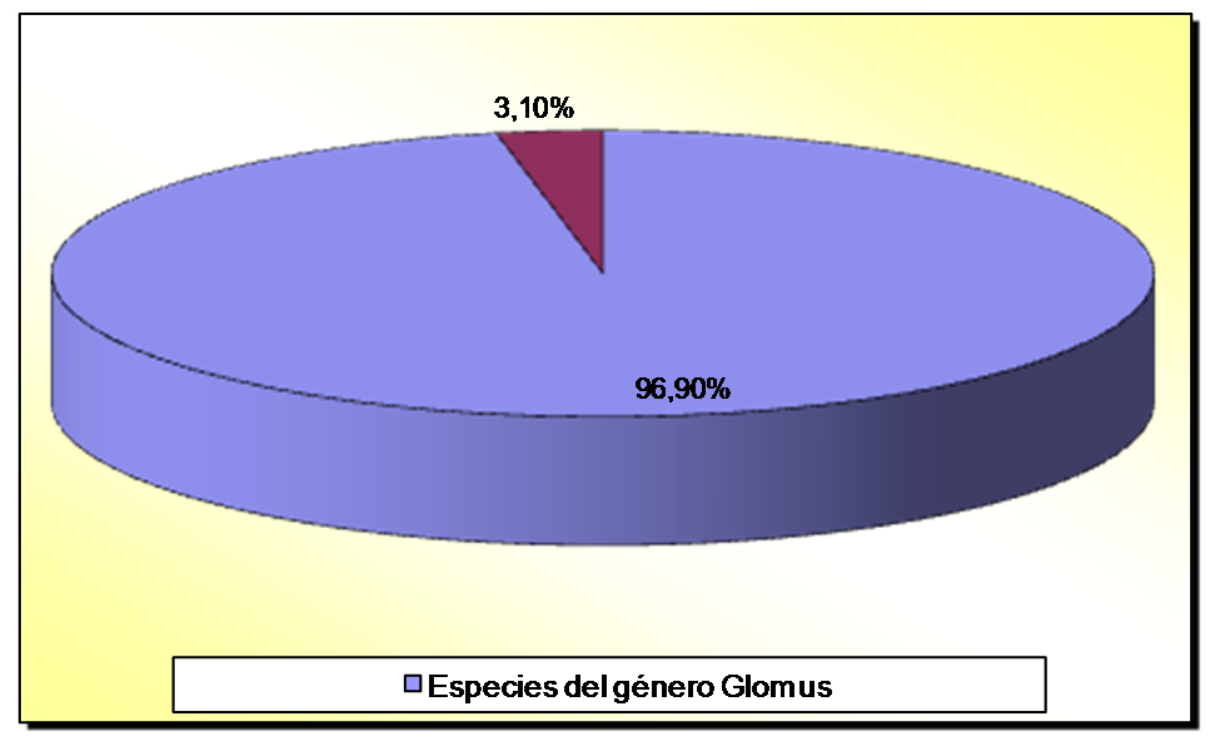

Figura 5. Distribución porcentual de géneros de hongos formadores de micorrizas arbusculares presentes en suelos ganaderos del municipio de Corozal. 
Con el modelo de función logística encontrado (Figura 4), se puede calcular las probabilidades de ocurrencia del evento ESPORULACION utilizando el vector de datos $\mathrm{X}$ originales, Por ejemplo, si se desea calcular cuál es la probabilidad de que ocurra esporulación en un suelo con un $\mathrm{pH}$ de 6.44 y un $\mathrm{K}$ de $0.16 \mathrm{meq} / 100 \mathrm{~g}$ de suelo, solamente se debe introducir los datos en la siguiente ecuación de la figura 6 y obtendremos el siguiente resultado mostrado en la figura 7.

$$
\hat{p}=\frac{e^{15.048-2.879^{*} P H+5.077^{*} K}}{1+e^{15.048-2.879^{*} P H+5.077^{*} K}}
$$

Figura 6. Ecuación para calcular las probabilidades de ocurrencia del evento ESPORULACION de hongos formadores de micorrizas arbusculares y valores de $\mathrm{pH}$ y K.

$$
\hat{p}=\frac{e^{15.048-2.879 * 6.44+5.077^{*} 0.16}}{1+e^{15.048-2.879 * 6.44+5.077^{*} 0.16}}=0.06=6 \%
$$

Figura 7. Cálculos de las probabilidades para las variables de $\mathrm{pH}$ y $\mathrm{K}$ y el evento de esporulación de Hongos formadores de micorrizas arbusculares en suelos de fincas ganaderas del municipio de Corozal.

Los resultados obtenidos en la ecuación, indicada en la figura 7, demuestra, que bajos los valores de $\mathrm{pH}$ y $\mathrm{K}$ anteriores se obtiene una probabilidad de ocurrencia del evento de esporulación de hongos formadores de micorrizas arbusculares en suelos rizosféricos de fincas ganaderas del municipio de Corozal en un $6 \%$. Con esta ecuación se obtienen, todos los cálculos de las probabilidades para todos los datos iníciales de las variables más importantes $\mathrm{pH}$ y K. La forma de utilizar este modelo consiste en introducir los valores de $\mathrm{pH}$ y $\mathrm{K}$ y calcular las probabilidades considerando significativas, aquellas probabilidades mayores del $50 \%$. Para el 
presente estudio se calcularon las probabilidades significativas, solamente para los valores de $\mathrm{pH}$ comprendido entre 4.6 y 5.8 y valores de $\mathrm{K}$ desde 0.4 hasta 0.5 , obteniéndose los valores de probabilidades desde $71 \%$ hasta $98 \%$ como se aprecia en la tabla 3.

Tabla 3. Valores ordenados de probabilidades utilizando el modelo definitivo (función logística) a partir de las variables continuas más importantes, pH y K.

\begin{tabular}{|c|c|c|c|}
\hline PH & K & ESPORAS & Probabilidad de esporulación \\
\hline 4,60 & 0,40 & 4721 & 0,98 \\
\hline 6,00 & 0,90 & 7230 & 0,91 \\
\hline 6,70 & 1,30 & 6041 & 0,91 \\
\hline 4,70 & 0,10 & 4730 & 0,88 \\
\hline 4,90 & 0,20 & 5617 & 0,88 \\
\hline 4,80 & 0,10 & 5662 & 0,85 \\
\hline 5,80 & 0,50 & 6496 & 0,71 \\
\hline
\end{tabular}

En la tabla 3 se muestran los valores de las probabilidades para cada valor de $\mathrm{pH}$ y $\mathrm{K}$, se observa que son significativos aquellos valores de probabilidad superiores al 50\%. Las variables más importantes que mejor explican el proceso de esporulación en el suelo, para las condiciones experimentales reportadas en el presente estudio, son en su orden: el pH y el K.

El grado de esporulación de hongos formadores de micorrizas arbusculares asociados con la especie (Bothriochloa pertusa) (L) A. Camus a través de parámetros químicos del suelo en fincas ganaderas, bajo las condiciones del municipio de Corozal, se puede evaluar de la siguiente manera: Para los valores de las variables métricas $\mathrm{pH}$ y $\mathrm{K}$ el modelo reducido que mejor representa la estructura de la relación entre $\mathrm{pH}, \mathrm{K}$ y la esporulación se pueden determinar por medio de la función logística mostrada en la figura 6. La utilización del modelo definitivo reporta que las mayores posibilidades de esporulación se presentan en suelos con características de $\mathrm{pH}$ desde "Fuerte a extremadamente ácida", 
"Moderadamente ácido", y "Ligeramente ácido" combinados con altos y medios niveles de potasio. La utilización del modelo, predice probabilidades de ocurrencia del evento esporulación que concuerdan completamente con las conclusiones de BAGYARAJ (1991); SÁNCHEZ (1998) y PEÑA (1999), con respecto a la compleja actividad del $\mathrm{pH}$ en el suelo y al hecho de que para la formación de hongos formadores de micorrizas arbusculares en suelos, se requiere un $\mathrm{pH}$ de 4.5 a 5.5 .

Los resultados anteriores, son procedentes y corroboran toda la literatura citada, en lo que respecta a la actividad compleja de los factores químicos en el suelo, con referencia a la esporulación. Todo esto concuerda con BAGYARAJ (1991), en el sentido de que en el suelo el efecto del pH es difícil de evaluar debido a que muchas propiedades químicas del suelo varían con el $\mathrm{pH}$, además se puede percibir que la naturaleza y estructura de las asociaciones entre estos factores químicos es tan compleja que amerita un estudio exploratorio con un mayor número de observaciones, un mayor número de zonas agroecológicas y un mayor número de variables como $\mathrm{Ca}, \mathrm{Al}, \mathrm{Na}, \mathrm{Mg}$, entre otros, así como la $\mathrm{CIC}$.

\section{Agradecimientos}

Los autores expresan sus agradecimientos, a la Universidad de Sucre por haber cedido sus laboratorios para el aislamiento e identificación de hongos formadores de micorrizas e igualmente a todos los ganaderos propietarios de las diferentes fincas ganaderas objeto del presente trabajo.

\section{Referencias.}

AGUILERA MARÍA. 2005. Economía Regional: La Economía del Departamento de Sucre: Ganadería y Sector Público. № 63, p. 54.

AHN-HEUM, E.; GAIL, W.; HARTNETT, D. 2001. Effect of ungulate grazer on arbuscular mycorrhizal simbioses and fungal community structure in tall grass prairie. Mycología 93 (2), p. $233-242$. 
AZCON, C. y BAREA, J. 1980. Micorrizas. En: Investigación y ciencia. V 47, p. 8.

AZCON, C. y BAREA, J. 1996. Interacciones de las micorrizas arbusuclares con microorganismos de la rizosfera. Micorrizas: Recurso Biológico del suelo, Fondo Fen en Colombia. Bogotá: Ediciones Eduardo Guerrero Forrero, p. 50.

BAGO, B.; AZCON, C.; PICHE, Y. 1998. Architecture and developmental dynamics of the external mycelium of the arbuscular mycorrhizal fungus glomus intraradices grown under monoxenic conditions. Mycología, 90 (1), p. 52.

BAGYARAJ, Joseph. 1991. Ecology of vesicular - arbuscular mycorrhizae. Handbook of applied mycología soil and plants. v 1, p. $13-14$.

BAREA , J. 1999. Importancia de las micorrizas arbusculares en el establecimiento y protección de las plantas en suelos degradados. Phytoma. Medio ambiente. Transferencia tecnológica, No. 111, p. 29.

BAREA, J. 2002. Rhizosphera and mycorrhizal of frield crops. Science and policy, p. 91.

BOLAN, N. 1991. critical review on the role of mycorrhizal in the uptake of phosphorus by plants. Plant and soil. p. $134-207$.

BONILLA B. 2000. Utilización de hongos micorrizógenos en la producción agrícola. CORPOICA. Boletín de investigaciones, v 3, p. 44.

BOTERO L., María. Influencia de las micorrizas arbusculares sobre el crecimiento del Aliso (Almus acuminata H.B.K. Subsp acuminata) en tres suelos diferentes de Colombia. Santafé de Bogotá, 1998, 90 p. Tesis (Maestría en Microbiología). Universidad de los Andes. Facultad de Ciencias. Departamento de Ciencias Biológicas.

CORWELL, W., BEDFORD, B., CHAPIN, C. 2001. Ocurrence of arbuscular mycorrhizal fungi in a phosphorus poor wetland and mycorrhizal response to phosphorus fertilization. American Journal of Botany 88 (10), p. 1824.

DANE. 2000. Encuesta Nacional Agropecuaria, Costa Atlántica, p. 16.

DANIELS, B.; SKIPPER, H. 1991. Methods for the recovery and quantitative estimation of propaqules from soil. En: SCHENCK, N. C. methods and principles of mycorrhizal search, APS Press. The American Phitopathological Society St. Paul. USA, p. $31-34$.

GONZÁLEZ, A. 1996. Las micorrizas como biofertilizantes en la agricultura. Curso cultivo e investigación del chontaduro, CORPOICA. 208 p. ISBN 958-9129-37-4. 
GUERRERO E. 1996. Micorrizas, recurso biológico del suelo. Fondo FEN de Colombia. Bogotá, 208 p. ISBN 958-9129-37-4.

HAIR, J. 1998. Multivariate Data Analysis. Prentice Hall. New Jersey. P 567.

HOSMER, J. y LEMESHOW, S. 1989. Applied Logistic Regression. John Wiley \& Sons, Inc. Canadá. P. 345.

INSTITUTO GEOGRAFICO AGUSTÍN CODAZZI. 1998. Estudio general de suelos y zonificación de tierras. Departamento de Sucre, p 1-10.

INTERNATIONAL COLLECTION OF ARBUSCULAR AND VESICULAR ARBUSCULAR MYCORRHIZAL FUNGI. 2002. Invam.caf.wvu.edu/mycinfo/taxonomy.

JEFFRIES, P. Y BAREA, J. 1999. Arbuscular Mycorrhiza a key component of sustainable plant - soil ecosystems. The mycota IX, fungal associations. Edition Hock, 113 p.

LUGO, M. y CABELLO, M. 2002. Native arbuscular mycorrhizal fungi (AMF) from mountain grassland (Córboba, Argentina), Seasonal variation of fungall spore diversity. Mycologia, 94(4), p. 579-586.

MENGE, J. A. and TIMMER, L. W. Procedures for inoculation of plants with vesicular arbuscular. Mycorrhizae in the Laboratory, Greenhouse, and Field. En: SCHENCK, N. C. Methods and principles of mycorrhizal research. APS Press, the American Phitopathological Society. Minnesota: Third printing, 1991. p. 59.

MORTON E. 1996. Classification and identification of mycorrhizal fungi, INVAM, Firs ICOM Workhosp. p. 1-5.

PEÑA L. 1999. Las micorrizas vesícula arbusculares (MA), una alternativa sostenible para los sistemas de producción de la zona andina de Nariño. CORPOICA. Boletín Técnico No. 9. 12 p.

PICONE Chris. 2000. Diversity and abundance of arbuscular - mycorrhizal fungus spores in tropical forest and pasture. Biotropica 32(4a), p. 734-750.

PLAN DE ORDENAMIENTO TERRITORIAL. 2002. Diagnóstico del Municipio de Sincelejo.

SALAMANCA C. 1999. Las micorrizas como estrategia de mejoramiento nutricional de pasturas y especies frutales en el departamento del Guaviare. Boletín Técnico CORPOICA No. 20, p. 1. 
SANCHEZ M. 1999. Endomicorrizas en agroecosistemas colombianos, Universidad Nacional de Colombia, sede Palmira, p. 33. ISBN 958-8095-01-8.

SCHENCK N. y PEREZ, Y.1990. Manual for the identification of VA mycorrhizal fungy. Tercera Edición. USA: Synergistic Publications, p. 17.

SIEVERDING, E. 1984. Aspectos básicos de la investigación de las micorrizas vesículo arbusculares. Investigación sobre micorrizas en Colombia. Memorias sobre el primer curso nacional sobre micorrizas. Universidad Nacional de Colombia. p. 9.

SIEVERDING, E. 1983. Manual de métodos para la investigación de las micorrizas arbusculares en el laboratorio. Centro Internacional de Agricultura Tropical, CIAT. p. 121.

SYLVIA, David. Mycorrhizal symbiosis. En: Sylvia, D; FUHRMANN, J.; HARTEL, P., and ZUBLERER, D. 1999. Principles and application of soil microbiology. Pretince Hall. New Jersey, p. 410.

TANG, F.; WHITE, J.; CHARVAT, I. 2001. The effect of phosphorus availability on arbuscular mycorrhizal colonization of typha angustifolia. En: Mycologia, 93 (6),. p. 1042.

TOTOLA, M. y CHAER, G. 2002. Microrganismos e processos microbiológicos como indicadores da qualidade dos solos. Sociedade Brasileira de Ciencias do solo, v 2, ISSN 1519-3934.

UNIVERSIDAD DE SUCRE. 1996. Situación actual del sector ganadero en el departamento de Sucre. Sincelejo, p. $5-65$.

VAN DER HEISDEN A.1998. Mycorrhizal fungal diversity determines plant biodiversity, ecosystem variability and productivity. Nature. Vol. 396, p. $69-72$. 